\title{
Marcus Sandl, Medialität und Ereignis. Eine
}

Zeitgeschichte der Reformation

Zürich : Chronos Verlag, 2011, 596 p., 55,50€

\section{Marion Deschamp}

\section{CpenEdition}

\section{Journals}

Édition électronique

URL : http://journals.openedition.org/ifha/7715

DOI : 10.4000/ifha. 7715

ISSN : 2198-8943

Éditeur

IFRA - Institut franco-allemand (sciences historiques et sociales)

Référence électronique

Marion Deschamp, « Marcus Sandl, Medialität und Ereignis. Eine Zeitgeschichte der Reformation », Revue de l'IFHA [En ligne], Date de recension, mis en ligne le 13 décembre 2013, consulté le 22 septembre 2020. URL : http://journals.openedition.org/ifha/7715 ; DOI : https://doi.org/10.4000/ifha.7715

Ce document a été généré automatiquement le 22 septembre 2020

(CIFHA 


\title{
Marcus Sandl, Medialität und Ereignis. Eine Zeitgeschichte der Reformation
}

\author{
Zürich : Chronos Verlag, 2011, 596 p., 55,50€
}

Marion Deschamp

La lecture de cette somme de près de 600 pages, résultant du mémoire d'habilitation de l'auteur, relèvera sans doute pour le lecteur français de la gageure, tant le niveau d'abstraction ici atteint est inhabituel, et disons-le d'emblée, confine parfois à l'abstrusion.

L'objet historique des triturations conceptuelles de M. Sandl est, pourtant, exemplairement ordinaire. Il s'inscrit dans le sillon des recherches allemandes et anglo-saxonnes sur l'histoire de la Réformation et plus particulièrement sur l'aspect médiatique du phénomène réformateur. Depuis les travaux pionniers de Bob Scribner, dans les années 1980, de nombreuses études se sont en effet consacrées à l'analyse des supports et ressorts médiatiques des partisans d'une réforme religieuse de l'Eglise romaine. L'étude des feuilles volantes, livrets, libelles ou brochures illustrées, a permis de reconstituer l'économie de diffusion et circulation des idées des partisans de la Réforme. D'autres études ont montré que l'importance du media imprimé n'a pas non plus diminué l'importance des régimes de communication oraux, parmi lesquels on comptait, en premier lieu, la prédication itinérante. Toujours est-il que les chercheurs font aujourd'hui chorus pour décrire, selon la formule de Johannes Burckhardt, la Réformation en termes de "révolution médiatique » (Reformation als Medienrevolution).

Mais si, au terme de ces décennies de recherche, la «chose» étudiée est restée relativement stable, les mots utilisés pour la décrire ont connus, eux, plus d'une valse sémantique. Tout se passe, en effet, comme si ce schème interprétatif de la diffusion de la Réforme se prêtait à un habillage théorique variant au gré des modes conceptuelles. Ces dernières, pour autant, n'offrent pas qu'un simple tour de passe-passe nominaliste : elles dévoilent au-delà de la labilité du vocable, de nouvelle manière de saisir l'objet historique, qui participent aussi de la construction du sens et d'un raffinement de l'interprétation des sociétés du passé. 
L'attirail conceptuel de Scribner, empruntant à la théorie de la communication de McLuhan et à la sémiologie moderne pour forger ses outils d'analyse (" propagande ", "hybridation des media», usage potentiel et effectif d'un signe, paradigme et syntagme, etc.), n'ont pas été retenus par ses successeurs, qui ont cependant conservé maints de ses résultats de recherche. Chassant, sur le marché conceptuel, les notions précédentes jugées trop empreintes d'un structuralisme désormais obsolète, un nouveau schème explicatif a fait son apparition à la fin des années 1990, imposant une autre nomenclature théorique. Soziale Interaktion, Kommunikationsraum, Vergesellschaftung, Medienereignis, Performativität und Selbstbeschreibung: ce lexique s'est mis alors à dominer l'écriture historique, sous la plume, entre autres, du Graduiertenkolleg de Giessen (Transnationale Medienereignis von der Frühen Neuzeit bis zur Gegenwart), et de l'école de Constance, emmenée par Rudolf Schögl. Ce dernier a construit son modèle analytique à partir, d'une part, des réflexions de Friedrich Kittler sur les technologies médiatiques, pensées comme consubstantielles aux structures sociales (à l'inverse de la thèse instrumentaliste de McLuhan); et, d'autre part, sur l'œuvre de Niklas Luhmann, et son analyse de la communication entre systèmes et subsystèmes sociaux. Appliqués à la compréhension des sociétés de la première modernité, et notamment au phénomène réformateur, ces nouveaux outils conceptuels ont souligné la fonction de catalyseur de l'imprimé et de la publicisation non seulement dans la diffusion des idées, mais surtout dans les processus de construction de groupes sociaux répondant au défi de la différentiation confessionnelle. Le caractère performatif et autocréateur d'identités individuelles et collectives a aussi été fortement souligné.

M. Sandl connaît bien les modèles théoriques développés par ces différents projets de recherche, pour y avoir lui-même participé, en tant que doctorant à Giessen, d'abord, puis wissenschaftlicher Mitarbeiter à Constance. Or, non seulement il manipule, dans son ouvrage, l'ensemble de ces concepts, mais semble vouloir encore y articuler le complexe théorico-sémantique échafaudé par sa nouvelle institution d'adoption, l'université de Zurich, qui l'a accueilli dans le cadre du projet «Medienwandel, Medienwechsel, Medienwissen ». Lancé en 2009 pour une période de 4 ans, le programme affiche rien moins que l'ambition d'établir, par l'étude des formes successives de perception, transmission et communication à travers les âges, une «médiologie historique " (historische Mediologie), c'est-à-dire une science historique des media. "Das Mediale ", ou "die Medialität » sont les contremarques conceptuelles des publications issues de ce programme, pour définir l'ensemble des conditions techniques de possibilités de médiation à une époque donnée.

L'ossature conceptuelle de Medialität und Ereignis est donc arcboutée sur ces différentes sources théoriques. Mais le lecteur, même connaisseur des évolutions et circonvolutions historiographiques sur le sujet, devra les deviner tout seul, tant le discours de M. Sandl reste obscur et évasif sur l'ensemble de ses références. D'ailleurs, on ne trouve, dans l'ensemble du livre, aucune explication claire des deux concepts éponymes. Plutôt que les définitions équationnelles classiques, M. Sandl privilégie les chiasmes sémantiques (Medien der Präsenz ou Präsenz der Medien, p. 65 ; Medienereignis und Ereignis als Medien, p. 218) ou les formules récursives (das Ereignishafte der Ereignis, p. 10), ou les deux à la fois («als apokalyptisches Ereignis korrelierte die Reformation (...) die Zeichenhaftigkeit der Wahrheit mit der Wahrheit der Zeichenhaftigkeit », p. 512). 
Finalement, la seule notion vraiment explicitée et qui semble servir au-delà d'une pratique de design théorique, est celle que l'auteur inscrit dans son sous-titre et aborde dans son premier chapitre: le temps. Celui-ci charrie avec lui une constellation d'abstractions: die Zeitgeschichte, die Zeitlichkeit, die Verzeitlichung, mais encore "die reformatische Episteme der Zeitenwende», "die immanente Zeit der Dirskurse», "die Poetologie historischer Zeiten", et, dans le sillage de Koselleck, die Deutungshorizont. Le projet de M. Sandl, celui d'écrire une histoire de la Réforme à partir de la vision qu'en eurent les contemporains (eine Zeitgeschichte der Reformation), nécessite selon lui de mettre à jour les conditions historiques changeantes d'appréhension du temps. Il s'agit donc de récuser l'idée d'un continuum temporel et de restituer les cadres intellectuels et descriptifs dans lesquels et par lesquels les hommes du XVIe siècle pensaient le déroulement du temps. Mais cela implique aussi de relire l'historiographie de la Réformation et ses découpages temporels (Spätmittelater, Reformation, konfessionnelles Zeitalter) de manière critique. L'analyse déconstructiviste des discours historiographiques se concentre en fait sur deux controverses classiques : la première s'identifie à l'appréciation de la Réforme luthérienne en terme de rupture ou de continuité avec l'époque lui ayant précédée, ce qui revient à soupeser la cohérence du phénomène réformateur comme séquence historique per se, et sa capacité à former une unité temporelle et événementielle. La deuxième consiste à interroger le potentiel de modernisation que la Réformation a suscité en Europe, et le rôle joué dans les processus de transformations politiques (étatisation), religieux (confessionnalisation, sécularisme) économiques (capitalisme) et sociales (discipline sociale).

Sur le premier point, M. Sandl remarque que la thèse de la rupture, soutenue au XIXe siècle par l'historien Leopold Ranke, et qui fit longtemps autorité, a perdu de son crédit dans les 40 dernières années, sous les coups de butoir conjoint des historiens spécialistes du Spätmittelalter, des défenseurs de la thèse de la confessionnalisation et, de manière différente (voire opposée) mais avec les mêmes effets, de l'approche microhistorique et de l'anthropologie religieuse (p. 31-33). En effet, pour les premiers, la théologie luthérienne manifestait une continuité et une congruence très forte avec maints courants de dévotion de la fin du XVe siècle, ne plaidant guère pour un changement radical de spiritualité offert par la Réformation. D'autre part, Heinz Schilling, et à sa suite les défenseurs du paradigme de la confessionnalisation, arguèrent que la Réformation n'avait rien d'une rupture révolutionnaire, mais constituait une étape dans un processus macrohistorique de modernisation se déroulant sur le long terme. Il en résultat une remise en cause res et verba de "la Réformation ». Enfin, les études microhistoriques mirent en exergue le caractère pluriel des idées, acteurs, et des mises en pratiques des principes réformateurs, ne permettant pas de parler d'un mouvement cohérent et unique. SANDL, pour sa part, cherche à sortir de cette dichotomie rupture/continuité, jugée aporétique. Sans récuser les thèses les plus récentes relativisant le caractère idiosyncratique de la Réformation, l'auteur entend restituer le discours des agents historiques eux-mêmes sur leur temps, qui selon lui, auraient vécu les décennies 1510-1530 comme un tournant historique (Zeitenwende). L'analyse interne des discours des acteurs permet à l'auteur de dévoiler les prémices de la tradition interprétative de la rupture. La Réformation, selon lui, à une raison d'être conceptuelle et historiographique, parce qu'elle se présenta ellemême comme événement historique. Certes, Sandl ne nie pas que le caractère performatif des énoncés du passé n'aille pas de soi. Mais il se range, en dernier lieu, à une compréhension interne des discours et dispositifs discursifs des agents historiques, 
qui se veut héritière de l'archéologie foucaldienne des discours. Et de privilégier trois prismes différents d'archéologie et fouilles textuelles, qu'il formule dans une triade conceptuelle (de son cru ?) : Selbstverhältnis des Diskurses, Selbstverhältnis des Medialen und Selbstverhältnis des Ereignishaften (p. 48-62).

Après ce lourd arsenal théorique, qui tient parfois du pot-pourri, M. Sandl déroule de manière logiquement chronologique les différents mouvements de sa démonstration. Dans les chapitres 2 et 3 , il relie la transformation, au début du XVIe siècle, de l'usage des media et l'effort de restauration de la vera doctrina dans un même élan d'évidence épistémologique. Dans le chapitre 4, il analyse les ego-documents et autres sources de la décennie 1520 se présentant comme des récits de conversion et mettant en scène l'évidence de la vérité à travers sa communication médiatisée. Le chapitre 5 , quant à lui, décortique l'obsession du tournant historique (Zeitenwende) qui se lit dans les sources contemporaines, et que l'auteur corrèle à la temporalité apocalyptique propre à la Réforme luthérienne. Le chapitre suivant se concentre sur l'examen de la confession d'Augsbourg, analysée comme moment de l'institution de la vraie tradition de l'Eglise. Quant aux deux derniers chapitres, ils étudient au plus près les modalités médiatiques d'écriture de l'histoire de la Réforme à partir des années 1530 et jusqu'à la rédaction du Livre de Concorde, en 1580. M. Sandl consacre un sous-chapitre particulièrement intéressant sur les premières biographies luthériennes, qui continuent post mortem l'autorité du prophète (p. 457-477). Pour M. Sandl, le mythe de la Réforme, unifiée autour de la figure charismatique de Luther, s'il tient de la fiction, n'en possède pas moins une force illocutoire, en fixant pour plusieurs siècles, un dispositif historique efficace d'interprétation du passé.

$\mathrm{Au}$ cours de ce long parcours, le lecteur courageux aura l'occasion de découvrir de nombreuses interprétations inspirées et intéressantes de grands textes du corpus réformateur. Les analyses que donnent $\mathrm{M}$. Sandl, notamment, des œuvres historiographiques de Flacius Illyricus, tel le Catalogus testis veritatis et les Centuries de Magdebourg, ou l'examen minutieux des controverses intraprotestantes sur l'enjeu de l'héritage luthérien, qui foisonnent entre l'Interim d'Augsbourg (1546) et la Formule de Concorde (1580), sont plutôt stimulantes sans être complètement originales. Le choix de l'auteur de se concentrer sur des textes extrêmement classiques et normatifs, écrits par les grandes figures de la Réforme (Luther en tête) est par contre plutôt décevante, eut l'égard de l'ambition de l'auteur d'appréhender l'histoire des temps de la Réforme par (l'ensemble de) ses contemporains. M. Sandl reproduit ici la tendance de certains historiens à généraliser l'expérience de la Réforme à partir d'un corpus balisé et qui force la cohérence rétrospective. On peut encore lui reprocher de se livrer, bien souvent, à une lecture herméneutique des sources qui ne se cantonne pas à en éclairer (avec brio) la logique immanente et la " poétologie ", mais (et c'est là son péché) à en dériver une efficacité intrinsèque sur les évènements du temps. C'est faire fi trop rapidement, nous semble-t-il des conditions socioculturelles de possibilité et d'efficacité des discours, bref, de ses conditions de félicité.

Pour finir, il est clair que la lecture de cet ouvrage apportera au lecteur courageux et désireux de se plonger dans les débats interprétatifs et théoriques les plus récents, des trésors de thèses à discuter. Qu'il ne s'attende pas cependant à être aidé par l'auteur, mais qu'il cherche plutôt à court-circuiter sa tendance hermétique tout en gardant ses efforts intellectuels. Hardi ! 
INDEX

Index chronologique : Frühe Neuzeit

Thèmes : Geschichte des Religiösen, Historiographie/Methodologie

\section{AUTEUR}

\section{MARION DESCHAMP}

Université Lumière Lyon 2 